\title{
Drainage, rebound and oscillation of a meniscus in a tube
}

Jeremy Marston, Garrett Toyofuku, Chao Li, Tadd Truscott, and Jamal Uddin

Citation: Physics of Fluids 30, 082103 (2018); doi: 10.1063/1.5038662

View online: https://doi.org/10.1063/1.5038662

View Table of Contents: http://aip.scitation.org/toc/phf/30/8

Published by the American Institute of Physics

\section{Articles you may be interested in}

Vibrational modes prediction for water-air bubbles trapped in circular microcavities

Physics of Fluids 30, 082001 (2018); 10.1063/1.5037328

A new experimental method based on volume measurement for determining axial scaling during breakup of drops and liquid threads

Physics of Fluids 30, 082102 (2018); 10.1063/1.5030330

Flow structure and evaporation behavior of an acoustically levitated droplet

Physics of Fluids 30, 082105 (2018); 10.1063/1.5037728

The hydrodynamic behavior of a squirmer swimming in power-law fluid

Physics of Fluids 30, 083301 (2018); 10.1063/1.5045701

Thermocapillary instability of self-rewetting films on vertical fibers

Physics of Fluids 30, 082104 (2018); 10.1063/1.5043482

Thermocapillary instabilities of liquid layers on an inclined plane

Physics of Fluids 30, 082101 (2018); 10.1063/1.5039149

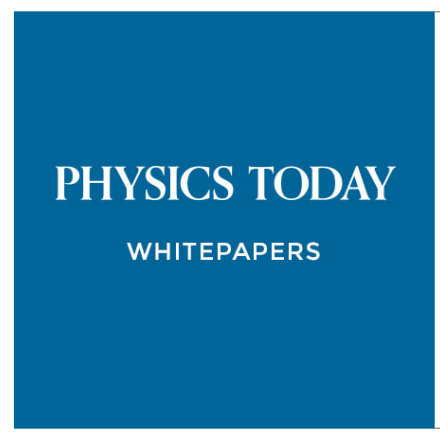

ADVANCED LIGHT CURE ADHESIVES

Take a closer look at what these environmentally friendly adhesive systems can do

\section{READ NOW}

PRESENTED BY

Q MASTERBOND。 


\title{
Drainage, rebound and oscillation of a meniscus in a tube
}

\author{
Jeremy Marston, ${ }^{1, a)}$ Garrett Toyofuku, ${ }^{1}$ Chao Li, ${ }^{1}$ Tadd Truscott, ${ }^{2}$ and Jamal Uddin ${ }^{3}$ \\ ${ }^{1}$ Department of Chemical Engineering, Texas Tech University, Lubbock, Texas 79409-3121, USA \\ ${ }^{2}$ Department of Mechanical and Aerospace Engineering, Utah State University, Logan, Utah 84322-4130, USA \\ ${ }^{3}$ School of Mathematics, University of Birmingham, Edgbaston B15 2TT, United Kingdom
}

(Received 4 May 2018; accepted 16 July 2018; published online 6 August 2018)

\begin{abstract}
In this paper, the drainage and subsequent rebound of a liquid column in a cylindrical tube is examined experimentally and theoretically. When liquid is drawn up into a capillary and then released under gravity, inertia allows the meniscus to overshoot the equilibrium capillary rise height. The meniscus then rebounds up the tube, again overshooting the equilibrium height and undergoes oscillation. By varying both the immersion depth and radius of the tube, one can observe rich dynamical behavior, with the most dramatic being the formation of a fast liquid jet, barely visible to the naked eye but easily captured with high-speed video. In addition to the flow separation caused by the sudden expansion at the end of the tube, this jet serves as a mechanism of energy dissipation. Some qualitative differences between the works of Quere et al. ["Rebounds in a capillary tube," Langmuir 15, 3679-3682 (1999)] and Lorenceau et al. ["Gravitational oscillations of a liquid column in a pipe," Phys. Fluids 14(6), 1985-1992 (2002)] and the present experiment are observed and discussed. A critical condition for oscillatory behavior is derived theoretically and matches well with the experimental observation. Once in the oscillatory regime, both the maximum depth below and the maximum rebound height above the equilibrium level are investigated by performing a systematic sweep through the relevant parameter space, incorporating the initial meniscus height, immersion depth, tube radius, and fluid properties. Lastly, the characteristic period of oscillation, $t_{p}$, is assessed and found to be largely independent of fluid viscosity, and could be reasonably well-collapsed by a single curve whereby $t_{p} \sim \sqrt{h_{i}}$, where $h_{i}$ is the tube immersion depth. Published by AIP Publishing. https://doi.org/10.1063/1.5038662
\end{abstract}

\section{INTRODUCTION}

The drainage of water in plastic straws is possibly one of the most easily accessible and reproducible fluid dynamics experiments and can be performed by scientists of all ages in any restaurant across the world. By immersing the straw in water, placing a finger tip over the top end and pulling the straw up, a column of water can be held in place and released at will by removing your finger tip. By carefully watching the ensuing motion, even the most novice of experimenters can observe that the water level will bounce a few times before settling. An overview of this rich dynamical process is presented in Fig. 1 for water in a glass tube of diameter $6 \mathrm{~mm}$. The liquid column is observed to drain steadily until it approaches its maximum depth below the equilibrium height and then rebounds up the tube and begins an oscillatory phase converging to the equilibrium height, marked by the red arrow.

The experiment reported herein is in some sense related to the dynamical capillary rise experiment, whereby a capillary tube dipped into water will draw liquid up, which is routinely used in undergraduate fluid mechanics to introduce surface tension. In this phenomenon, the balance of surface tension forces, $F_{\sigma}=2 \pi R \sigma \cos \theta$, and the weight of the liquid column, $F_{g}=\pi R^{2} \rho h_{e} g$, yields the equilibrium capillary rise height

$$
h_{e}=\frac{2 \sigma \cos \theta}{\rho R g},
$$

a)Electronic mail: jeremy.marston@ttu.edu where $\sigma$ is the surface tension, $\theta$ is the contact angle between the tube interior and the meniscus, $\rho$ is the liquid density, $R$ is the tube radius, and $g$ is acceleration due to gravity. As a first approximation, the dynamics of attaining this equilibrium for an incompressible fluid in a long cylindrical pipe could be described by Newton's second law (e.g., Ref. 14) involving surface tension, viscous and gravitational forces as

$$
\rho \frac{d}{d t}(h \dot{h})=\frac{2 \sigma \cos \theta}{R}-\frac{8 \mu}{R^{2}} h \dot{h}-\rho g h,
$$

and, for a tube dipped into liquid, is subject to the initial conditions $h(0)=0$ and $\dot{h}(0)=0$, where the dot denotes a time-derivative. We note that this ODE does not explicitly deal with oscillatory motion and assumes Poiseuille flow throughout the tube. The dynamics of this situation have been studied, among others, by Refs. 6, 14, 5, 9, 3, 2, and 13, and can essentially be characterized by three asymptotic regimes: (i) pure acceleration with $h \sim t^{2}$, (ii) linear regime with $h \sim t$, and (iii) quasi-steady regime with $h \sim t^{1 / 2}$. Note that in regime (iii), the Lucas-Washburn equation is recovered, which has the short-time asymptotic solution

$$
h(t)=\sqrt{\frac{R \sigma \cos \theta}{2 \mu} t},
$$

which can be readily compared to experimental data, but has been subject to scrutiny for the fact that there is a singularity in velocity as $t \rightarrow 0$ (Ref. 14). However, it is in regime 


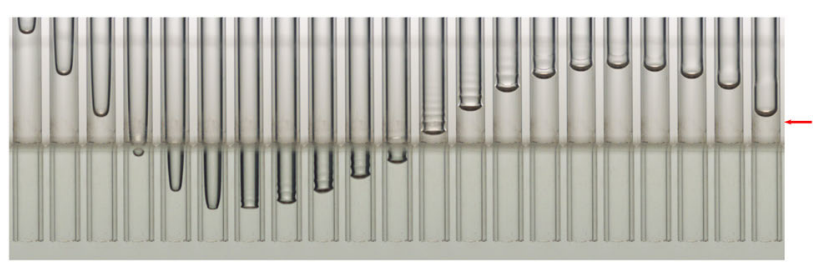

FIG. 1. A water meniscus falling and rebounding in a partially submerged glass tube with inner diameter of $6 \mathrm{~mm}$. The immersion depth is $23 \mathrm{~mm}$ and the initial fill height above the free surface was $97 \mathrm{~mm}$. The red arrow indicates the location of the equilibrium capillary rise for this configuration. See also video 1 of the supplementary material accompanying this figure.

(i) with the modified initial condition of $h(0) \neq 0$ that perhaps the most interesting behavior associated with this phenomenon occurs-namely_bouncing or oscillation, which was reported in experiments by Quere ${ }^{10}$ and Lorenceau et $_{\text {al. }}{ }^{7}$ In their experiments, a tube filled with air was submerged in liquid and the meniscus motion up the tube was initiated by the hydrostatic pressure. The meniscus was observed to overshoot its equilibrium and to undergo multiple oscillations before becoming quiescent. Theoretical treatment of the problem by Refs. 10 and 7 involved energy considerations resulting in a cubic equation for the height of the meniscus, which compared favorably to experimental data. The oscillatory behavior was also neatly explained by Zhmud et al. ${ }^{14}$ by considering the solution to Eq. (2) in the limit of negligible viscous effects with a modification to allow for both upward and downward motion. If one considers the initial meniscus height, $h(0) \neq h_{e}$ due to the filling of the tube as a perturbation from the equilibrium height, then the height can be described by $h(t)=h_{e}$ $+\epsilon(t)$. Upon substitution into (2), it can then be shown that the perturbation $\epsilon(t)$ is described by a harmonic motion with frequency $\sqrt{g / h_{e}}$.

The numerical investigation by Masoodi et al..$^{9}$ solved the governing equation with an emphasis on the oscillatory behavior; by non-dimensionalization of Eq. (2), a dimensionless parameter, $\omega$, was introduced with

$$
\omega=\frac{\rho^{2} g R^{4}}{64 \mu^{2} h_{e}} \equiv \frac{1}{128 \cos \theta}\left(\frac{B o}{O h}\right)^{2},
$$

where $B o=\frac{\rho g R^{2}}{\sigma}$ is the Bond number and $O h=\frac{\mu}{\sqrt{\rho R \sigma}}$ is the Ohnesorge number. It was tentatively concluded that this parameter governs oscillatory behavior, whereby oscillation occurred only for $\omega>\omega^{*} \approx 0.25$. The model of Masoodi et al. ${ }^{9}$ was also able to correctly describe the oscillatory behavior that was observed experimentally by Quere et $a l .{ }^{10}$ and Lorenceau et al. ${ }^{7}$

To the authors' knowledge, however, there is only one study of the inverse experiment (Ref. 12) to that of Quere et al. ${ }^{10}$ and Lorenceau et al. ${ }^{7}$ i.e., where the meniscus begins far above the equilibrium height $\left(h(0) \gg h_{e}\right)$. In that work, experimental results for two tube diameters $(6.2$ and $25.4 \mathrm{~mm})$ compared favorably to numerical solutions of the governing equations, however, the influence of fluid viscosity and critical conditions for oscillations were not assessed. In addition, there have not been any experimental studies which have systematically studied the oscillation period and amplitude as a function of the governing parameter, $\omega$. Furthermore, no experimental validation of $\omega^{*}$ exists. These three observations thus form the focal points of the present study, with the principal result being validation of $\omega^{*}$ for the case where $h(0) \gg h_{e}$. We begin in Sec. II by recapitulating the theoretical model (e.g., Refs. 10 and 9), which captures the oscillatory behavior; Sec. III presents an overview of the experimental setup and methods; Sec. IV presents the experimental data and comparison to theory, whilst in Sec. V we show some interesting local features of the phenomena such as wave focusing and jetting. Finally, we conclude the paper in Sec. VI with a discussion of the present study and suggestions for further work.

\section{THEORY}

We start with Eq. (1), and follow the analysis of Quere et al., ${ }^{10} \mathrm{Zhmud}$ et al.,${ }^{14}$ and Masoodi et al. ${ }^{9}$ to account for upward and downward motion. Essentially this entails modification of the momentum term on the left-hand side of Eq. (2), and we refer the reader to those papers for discussion thereof. We then non-dimensionalise such that heights are made dimensionless with respect to $h_{e}=\frac{2 \sigma}{\rho g R}$ and times are scaled with $\tau=\frac{8 \mu h_{e}}{\rho g R^{2}}=\frac{8 \mu \cdot 2 \sigma}{\rho^{2} g^{2} R^{3}}$, then we have (similar to Ref. 9) the following dimensionless equation:

$$
\omega\left(H \ddot{H}+\frac{1}{2}\left(\dot{H}^{2}+\dot{H}|\dot{H}|\right)\right)=1+\alpha-H-H \dot{H},
$$

where $\omega$, defined in Eq. (4) can be written as $\omega=\frac{\rho^{3} g^{2} R^{5}}{128 \sigma \mu^{2}}$, $H=h / h_{e}$, and $\alpha=h_{i} / h_{e}$ where $h_{i}$ is the immersion depth of the tube [as defined in Fig. 2(a)]. The second term on the left-hand side, $\dot{H}^{2}+\dot{H}|\dot{H}|$, deals with the upward and downward motion, whereby this quantity is obviously zero for negative velocity. Note also that $\tau=\sqrt{g / h_{e}} \sqrt{\omega}$.

For our case, we consider $H=(1+\alpha)+\epsilon z$ with $\epsilon \ll 1$. When we substitute this into Eq. (5) we have

$$
\omega \ddot{z}+\dot{z}+\frac{1}{(1+\alpha)} z=\epsilon F(z, \dot{z})
$$

If we consider only leading order terms (i.e., ignore the right hand side above), then we have the following second order ordinary differential equation (ODE):

$$
\omega \ddot{z}+\dot{z}+\frac{1}{(1+\alpha)} z=0
$$

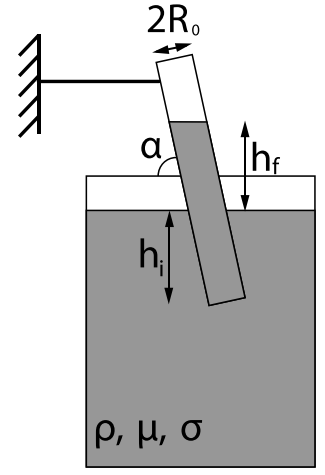

(a)

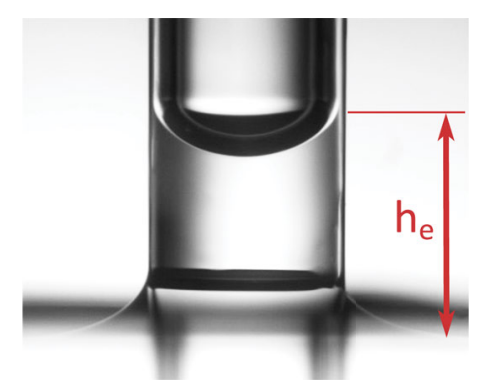

(b)
FIG. 2. (a) Schematic representation of the experimental configuration. (b) Photograph of the equilibrium capillary rise of water in a $R=2 \mathrm{~mm}$ tube. 
which has the solution

$$
z=\exp \left(-\frac{T}{2 \omega} \pm \frac{T}{2 \omega} \sqrt{1-\frac{4 \omega}{(1+\alpha)}}\right)
$$

In order to have oscillations we require the term in the square root to be negative, which imposes the following inequality:

$$
\omega>\frac{(1+\alpha)}{4} \text {. }
$$

When $\omega$ satisfies this condition we have

$$
z=\exp \left(-\frac{T}{2 \omega}\right) \cos \left(\frac{T}{2 \omega} \sqrt{\frac{4 \omega}{(1+\alpha)}-1}\right)
$$

from which it is obvious that the motion is periodic but decays over time. Thus the half-period of oscillation (as defined in the experiments—-see Sec. IV) is given by

$$
T=\frac{2 \pi \omega \sqrt{(1+\alpha)}}{\sqrt{4 \omega-(1+\alpha)}} .
$$

In dimensional terms this is given by

$$
t_{p}=\frac{2 \pi \omega \sqrt{h_{e}(1+\alpha)}}{\sqrt{g \omega} \sqrt{4 \omega-(1+\alpha)}} .
$$

Using an example from Fig. 4 for a tube with inner diameter $2 \mathrm{~mm}$, an immersion depth of $h_{i}=46 \mathrm{~mm}, \rho \approx 10^{3} \mathrm{~kg} / \mathrm{m}^{3}$, $R=1 \mathrm{~mm}, \theta \sim 0$ and $\sigma=0.072 \mathrm{~N} / \mathrm{m}$, we have $\alpha \approx 4$. In this case we have $\omega \approx 10.8$. We note that $\omega$ is indeed greater than $(1+\alpha) / 4=1.25$ required for oscillations to take place. Also in this realization we calculate the half-period of oscillation to be

$$
t_{p}=\frac{2 * 3.14 * 10.8 * \sqrt{0.014 * 5}}{\sqrt{9.8 * 10.8} \sqrt{43.2-5}}=0.282 \mathrm{~s} .
$$

This is in good quantitative agreement with the experimentally determined values, in Figs. 6 and 7, discussed later. To quantitatively compare the above analysis to the experiments, we solve Eqs. (5) and (11) using the experimental parameters. The results of this approach are discussed in Sec. IV.

\section{EXPERIMENTS}

The experiment consists of a glass tube which is immersed in liquid to a depth of $120 \mathrm{~mm}$ and then retracted to achieve a specified initial meniscus height, $h_{f}$, and immersion depth, $h_{i}=120-h_{f}$. We used several immersion depths in increments of $\Delta h_{i} \approx 10 \mathrm{~mm}$ to achieve a range of fill-to-immersion ratios $h_{f} / h_{i} \approx 0.8-25$. The glass tubes have circular cross-sections with inner diameters $D=2 R=2,4,6,8$ and $10 \mathrm{~mm}$ and length of $30 \mathrm{~cm}$.

The tank into which the tubes are immersed has a crosssection $20 \times 20 \mathrm{~cm}^{2}$ and the water level was kept constant at $12 \mathrm{~cm}$. Thus the cross-sectional ratio between the tubes and the tank was small in all cases. In particular, The distance between the tube and the tank wall was at least 7.8 tube diameters. The glass tubes were clamped into position using an optical rail and adapters and a flexible hose was connected to the top of each glass tube in order to achieve an easy and repeatable release of the liquid column. A schematic representation of the setup is shown in Fig. 2(a). The majority of the experiments were performed with the tubes oriented vertically $\left[\alpha=90^{\circ}\right.$ in
TABLE I. Physical properties of the liquids used in the experiments. The glycerol mixtures are stated as concentration by volume.

\begin{tabular}{lccc}
\hline \hline Liquid & $\begin{array}{c}\text { Viscosity, } \\
\mu(\mathrm{mPa} \mathrm{s})\end{array}$ & $\begin{array}{c}\text { Density, } \\
\rho_{l}\left(\mathrm{~kg} / \mathrm{m}^{3}\right)\end{array}$ & $\begin{array}{c}\text { Surface tension, } \\
\sigma(\mathrm{mN} / \mathrm{m})\end{array}$ \\
\hline Pure water & 1.0 & 996 & 72.1 \\
$2 \%$ w $w$ SDS & 1.0 & 996 & 38.0 \\
$25 \%$ glycerol & 2.3 & 1074 & 71.0 \\
$50 \%$ glycerol & 8.1 & 1146 & 69 \\
$60 \%$ glycerol & 14.2 & 1169 & 68.5 \\
$70 \%$ glycerol & 30.54 & 1194 & 67.8 \\
\hline \hline
\end{tabular}

Fig. 2(a)], but some trials were performed at angles $\alpha=30^{\circ}$ and $45^{\circ}$.

The entire drainage, rebound and oscillation for each trial were captured in a single video recording using a high-speed video camera (Phantom Miro 310, Vision Research Inc. or Fastcam Mini UX-100, Photron Ltd.) with a manual trigger. Backlighting rendered silhouette-type imaging which yielded good contrast between the background and the meniscus, thus enabling facile tracking of the water-air interface (i.e., meniscus) using commercial tracking software (Photron Fastcam Analysis). Frame rates between $500 \mathrm{fps}$ and $10000 \mathrm{fps}$ were used to capture the dynamics with sufficient temporal resolution. A $55 \mathrm{~mm}$ Nikon micro-Nikkor lens yielded effective pixel sizes down to $47 \mu \mathrm{m}$ per pixel. Thus our uncertainty in the location of the meniscus is $\pm 47 \mu \mathrm{m}$.

The fluids tested comprised pure water, water-glycerol mixtures and a surfactant solution (Sodium Dodecyl Sulfate), whose concentrations and physical properties are tabulated in Table I.

Still images of the undisturbed capillary rise height, denoted herein as $h_{e}$, were taken for reference [see for example Fig. 2(b)] and we assume the sign convention of positive for heights above this level and negative for heights below this. Note that in many cases, the first overshoot is actually below the free-surface of the liquid in the tank and below the end of the tube. In this case, jetting can arise from the collapse of the air cavity that protrudes into the tank, which is discussed in Sec. V.

\section{OSCILLATIONS}

As a qualitative overview of the influence of both tube radius and immersion depth, we present to the reader Figs. 3 and 4. The image sequences shown in these figures are representative of typical video recordings from which we can extract quantitative measurements-namely - the meniscus location, $h(t)$, and the time between successive maxima and minima (i.e., the half-period of oscillation, $t_{p}$ ). The images in these sequences are taken from approximately the same reference time so one can visually gauge the effect of changing the tube radius (Fig. 3) and immersion depth (Fig. 4). From a simple comparison of these two figures, one could say that varying the immersion depth (for fixed radius) has a stronger effect than varying the tube radius (for fixed immersion depth), fluid properties notwithstanding. However, we should keep in mind that the interaction of these two parameters comes into play, 


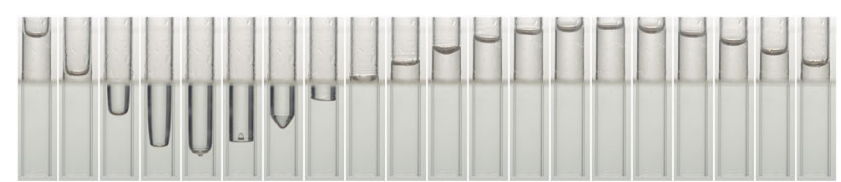

(a)

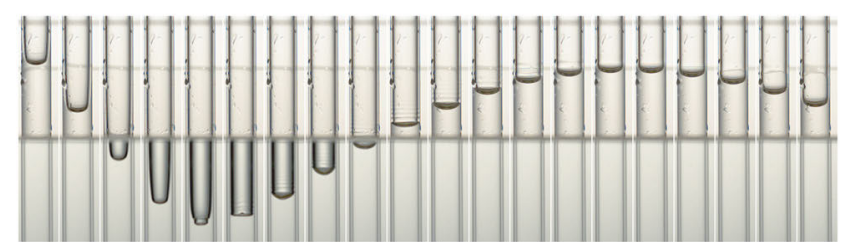

(b)

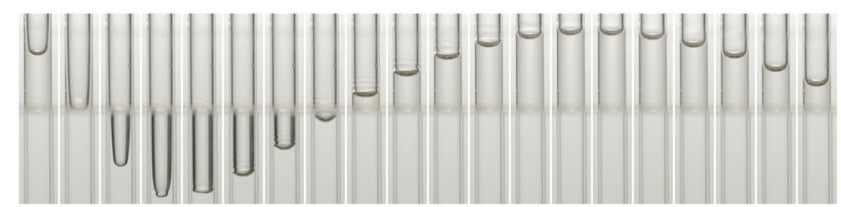

(c)

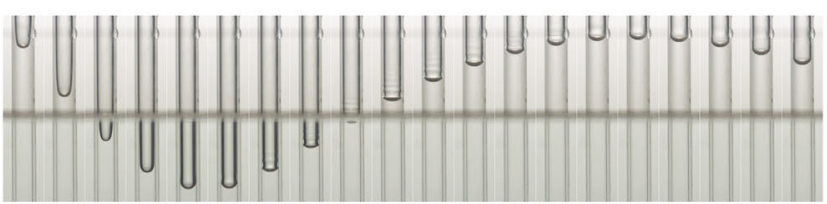

(d)

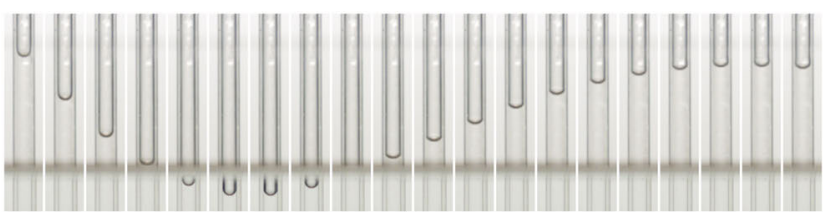

(e)

FIG. 3. Image sequences from high-speed video recordings of the first rebounds of a water meniscus in tubes of radii $R=5,4,3,2$ and $1 \mathrm{~mm}$. The immersion depth in each case was $h_{i}=36 \mathrm{~mm}$ and the fill height above the free-surface, $h_{f}=84 \mathrm{~mm}$. The frames are each separated by $d t=20 \mathrm{~ms}$. The corresponding values of $\omega$ and $\alpha$ are: $\omega \approx 32$ 632, 10 692, 2537, 334, and $10.4 ; \alpha \approx 12.3,9.81,7.36,4.91,2.45$.

which is evident if we consider Eq. (5), which contains the non-dimensional parameter $\alpha$, which is related to the product of these two factors, i.e., $\alpha=h_{i} / h_{e} \sim h_{i} R$.

\section{A. Initial acceleration}

The initial acceleration of the meniscus immediately after release is shown in Fig. 5(a) for a range of tube diameters, all from an initial height of $h_{f}=82 \pm 2 \mathrm{~mm}$ above the free-surface $\left(h_{f} / h_{i}=2.16\right)$. The black dash-dot line indicates the pure free-fall trajectory of a particle under gravity, i.e., $z(t)=\frac{1}{2} g t^{2}$, thus indicating that viscous and capillary forces play some role in the initial drainage of the tube, even for diameters larger than the capillary length, $l_{c}=\sqrt{\sigma /(\rho g)} \approx 2.7 \mathrm{~mm}$. Clearly, the largest deviation from free-fall is with the smallest capillary diameter, $R=1 \mathrm{~mm}$, where $2 R<l_{c}$. We expect that the dynamics will be most affected by capillarity for tube diameters smaller than the capillary length, whereas for the larger tubes with $R \geq 2 \mathrm{~mm}$, we expect this influence to diminish. As per Eq. (2), we see that the magnitude of the viscous term scales with radius as $\sim 1 / R^{2}$, whilst surface tension scales with $\sim 1 / R$ so that both contributions become increasingly significant as the tube radius decreases. The

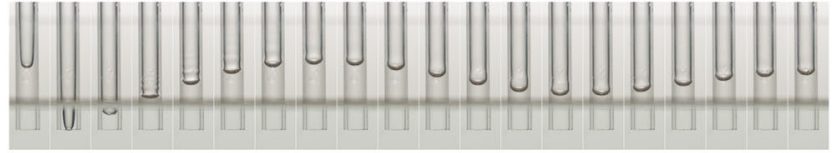

(a)

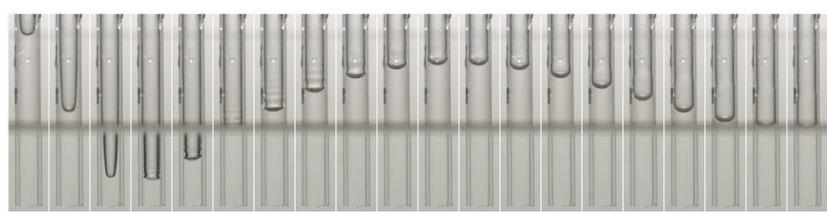

(b)

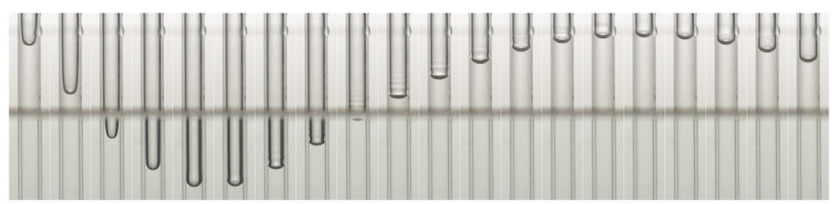

(c)

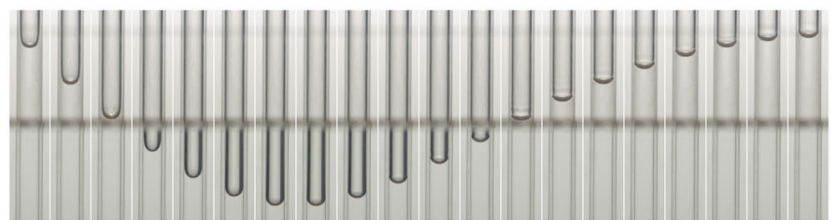

(d)

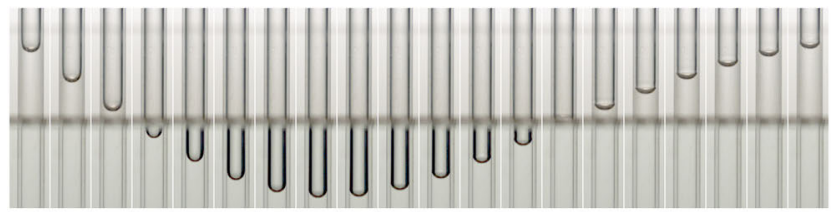

(e)

FIG. 4. Image sequences from high-speed video recordings of the first rebounds of a water meniscus in a tube of radius $R=2 \mathrm{~mm}$, giving $\omega=334$. The immersion depths are $h_{i}=6,16,36,56$, and $66 \mathrm{~mm}$ and the fill heights above the free-surface, $h_{f}=114,104,84,64$, and $54 \mathrm{~mm}$. The frames are each separated by $d t=20 \mathrm{~ms}$. The corresponding values of $\alpha \approx 0.82,2.18$, $4.91,7.63$ and 8.99 .

initial motion is important to keep in mind when discerning between trials in the oscillating regime because the speed of the meniscus as it passes through equilibrium essentially determines the magnitude of the "overshoot," as discussed below.

A comparison between experiment and theory, based on numerical integration of Eq. (5) is shown in Fig. 5(b). For clarity, we focus only on the largest and smallest tube radii; There is reasonable agreement in both cases, however, we note that the fit for $R=5 \mathrm{~mm}$ is better than that for $R=1 \mathrm{~mm}$. Again, this highlights the significance of capillary effects. In Eq. (5), capillarity is captured by both the coefficient $\omega$ and the term $\alpha=h_{i} / h_{e}$, yet it appears that the model slightly underestimates the true magnitude of the effect.

\section{B. Influence of immersion depth}

An example of the raw data extracted from the video sequences is presented in Fig. 6(a) for $R=1 \mathrm{~mm}$. The plotted lines represent the location of the meniscus relative to the equilibrium height, $h(t)-h_{e}$. For conciseness, we plot only the region in the vicinity of the equilibrium, i.e., $h_{e} \pm 15 \mathrm{~mm}$, which encompasses the maximum penetration and rebound for 


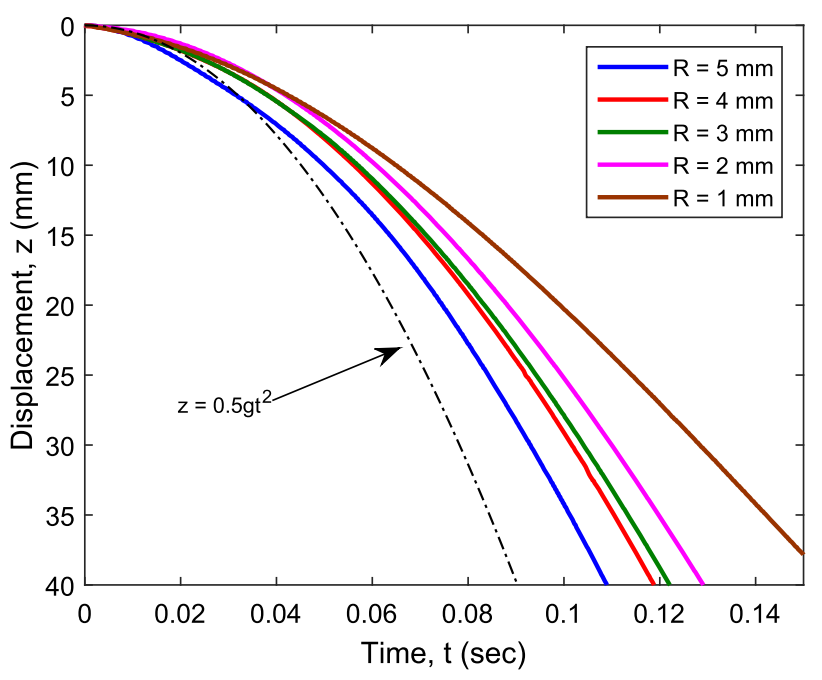

(a)

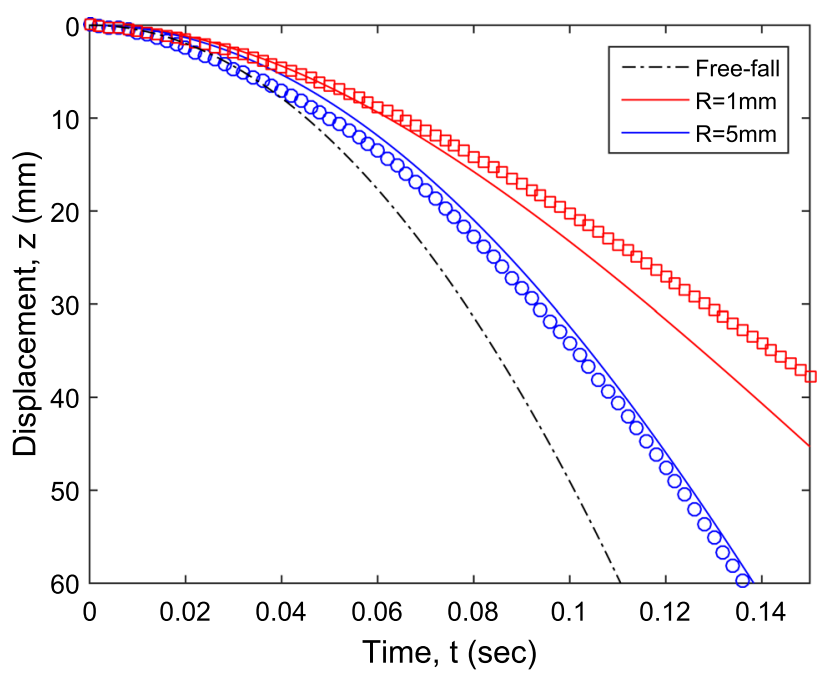

(b)

FIG. 5. (a) Displacement-time curves at the beginning of motion of a water mensicus in tubes of $R=1,2,3,4$ and 5 mm. The colored data points are the experimental data whilst the black dash-dot line represents the pure free-fall solution, $z(t)=\frac{1}{2} g t^{2}$. (b) Comparison between experiment (open symbols) and numerical solution (solid lines) to Eq. (5); The solution free-fall equation is shown by the black dash-dot line.

all the different immersion depths for this tube. Although such oscillations can continue for some time ( $t>60 \mathrm{~s}$ in Ref. 7$)$, the amplitudes diminish significantly for our experimental configuration after approximately $2 \mathrm{~s}$ to within $1 \mathrm{~mm}$ of $h_{e}$. From the trajectories in Fig. 5, it is clear that the early meniscus velocity is a function of the tube radius. However, we also observe that it is a function of the fill-to-immersion ratio. For example, with reference to Fig. 6(a), if we extract the approximate velocity of the meniscus as it first passes the equilibrium height, $h_{e}$, we find a monotonic increase in velocity $\left.V\right|_{h_{e}}: 0.16 \rightarrow 0.48 \mathrm{~m} / \mathrm{s}$ as $h_{f} / h_{i}: 0.82 \rightarrow 17.4$.

Interestingly, the minimum height below the equilibrium, i.e., the first local minima in the curves during exhibits a nonmonotonic dependence upon the initial fill height, or equivalently, on the velocity $\left.V\right|_{h_{e}}$. In particular, we observe that the miminum height reached for $h_{f} / h_{i}=17.4$ is almost the same as that for $h_{f} / h_{i}=0.82$, around $-10 \mathrm{~mm}$. However, for intermediate values, the meniscus penetrates deeper with the deepest penetration around $-15 \mathrm{~mm}$ for $h_{f} / h_{i}=2.3$. This implies that there is an optimum fill-to-immersion ratio which yields the maximum amplitude of oscillation (i.e., deepest penetration and highest rebound), given by an intricate balance of the energies and loss mechanisms. In other words, the maximum amplitude is a trade-off between the momentum of the liquid in the tube, $\sim \frac{d}{d t}(h \dot{h})$, and the energy loss due to the sudden expansion at the end of the tube, which one expects to scale as $\sim \dot{h}^{2}$, capillary and viscous forces notwithstanding.

In Fig. 6(b), we plot the numerical solution of Eq. (5) for the same cases as in Fig. 6(a). The main plot shows the full motion from the moment of release, whilst the inset graphic shows the zoomed region around the first few oscillations. We find that the salient features of the phenomena are welldescribed by the theory. In particular, the qualitative trend of a maximum penetration depth for intermediate immersion depths is captured. At a quantitative level, the maximum depths slightly over-predict the experimental values, with the largest discrepancy observed for $h_{i}=6 \mathrm{~mm}$.
For a direct comparison of experiment and theory, Fig. 7 shows four specific cases from the data of Fig. 6 for $h_{i}=66,46,27$, and $6 \mathrm{~mm}$. To enable the comparison, the theory curves have been shifted to account for the fact that the experimental data starts from the first frame the meniscus enters the field-of-view. One clear trend is that as the immersion depth decreases, the theory over-predicts the meniscus penetration. Overall, however, the comparison is very favorable and indicates that the theory describes the data very well and captures the salient features of the phenomena.

\section{Maximum penetration, PIV analysis and energy loss}

The maximum penetration and maximum rebound observed in Fig. 6 was found to be a consistent feature across the range of tube radii used herein and is best visualized by plotting the absolute maximum depth or rebound height against the fill-to-height ratio, $h_{f} / h_{i}$. This data is presented in Fig. 8(a) for water, where the data points shown are relative to the equilibrium level. It is clear that all data sets follow a nonmonotonic trend, i.e., they all exhibit a distinct local minima in the minimum depth (squares), which occurs at $h_{f} / h_{i}=2.3$ for $R=1 \mathrm{~mm}$, but for all other diameters, occurs at approximately $h_{f} / h_{i} \approx 1.5 \pm 0.2$. This trend is mirrored in the data for the maximum rebound height (circles), which all exhibit a local maxima at the same value of $h_{f} / h_{i}$, as one would expect given the greater overshoot from equilibrium. Figure 8(b) shows the specific case for water with $R=5 \mathrm{~mm}$ using a finer resolution sweep through the parameter space of immersion depth; This plot reveals the true maximum penetration of $-32 \mathrm{~mm}$, which occurs at $h_{f} / h_{i}=1.67$. Similar qualitative trends are observed for the other liquids, and we can rationalize these observations by considering the energy loss and vortex structure as fluid exits the straw.

Performing particle image velocimetry (PIV) measurements at the exit of the different straws show that the largest portion of flow field dissipation occurs during the first rebound. 


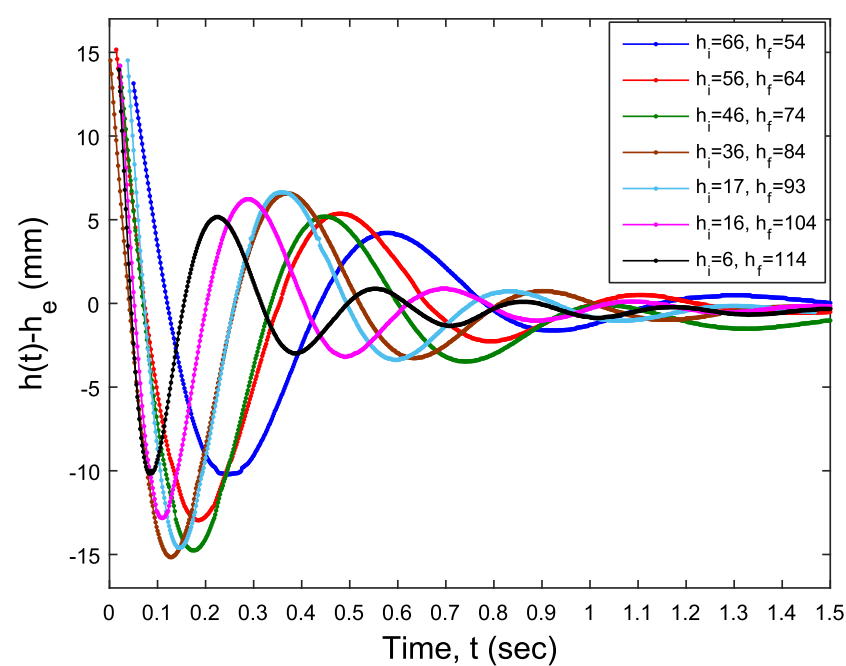

(a)

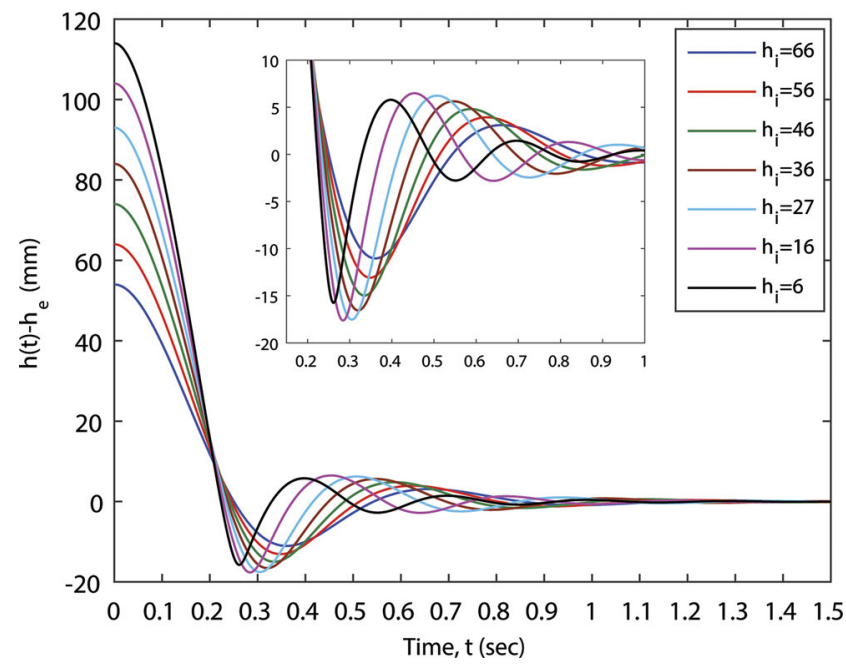

(b)

FIG. 6. (a) Time-resolved location of the meniscus apex plotted relative to the equilibrium height, $h_{e}$, for water in a tube with $R=1 \mathrm{~mm}(\omega \approx 18)$. The legend indicates the immersion depths of the tube, $h_{i}$. (b) Numerical solution to Eq. (5) for water for the same cases shown in (a). The main plot indicates the full motion from the moment of release, whilst the inset shows the zoomed region around the first few oscillations.

Rebound vortices occurring after the first instance show very little vorticity and indicate that a large portion of the flow energy dissipates during the first rebound. Figure 9 shows PIV data for four different cases. Each of the cases was analyzed using a multipass PIV algorithm in exactly the same way with a $64 \times 64$ pixel and then a $32 \times 32$ pixel window with $50 \%$ overlap. The arrows in the figure represent the velocity vectors where $1 \mathrm{~m} / \mathrm{s}$ is 10 pixels. Colors represent the z-vorticity $(1 / \mathrm{s})$ in the flow field as shown in the colorbar. The mask at the top (red box) represents where the straw was in the flow field and the masks are 84 pixels across or $4 \mathrm{~mm}$ outside diameter.

Each of the tubes were filled to $120 \mathrm{~mm}$ and then submerged to the depths revealed in the caption. The maximum vorticity occurs shortly after the release of the fluid, when the maximum displacement has occurred for all cases. The first vortex released contains the majority of the energy from the motion of the rebounding fluid, while the second vortex is similar and much smaller for all cases (as confirmed by Fig. 10). The deeper the immersion depth the longer the duration between vortex shedding. This makes sense as the deeper immersion depths means there is a larger hydrostatic pressure force opposing the rebound. At these small tube radii the second rebound is nearly at the end of the rebounds, but even then it is clear that the second vortex release is on the same order of magnitude for all the immersion depths.

To futher assess the energy loss at successive rebounds, we present to the reader Fig. 10, which plots the percentage energy loss, calculated from the change in potential energy from successive maxima in the meniscus height, i.e., $\Delta E_{i, \%}$ $=100 \times\left(1-h_{\max , i+1} / h_{\max , i}\right)$. In agreement with the PIV observations, we clearly see the largest energy loss at the first rebound, and that this energy loss is inversely related to the immersion depth. The loss at the first rebound, $\Delta E_{1}$ ranges from $50 \%$ to $80 \%$, whereas the range for successive rebounds diminishes rapidly, whereby $\Delta E_{3} \approx 5 \%$, and $\Delta E_{4} \approx 1 \%-2 \%$. These observations are easily understood from established fluid mechanics of head loss at reentrant pipe inlets/exits, from which we can establish the energy loss dependence as $\Delta E \propto \dot{h}^{2}$, which therefore explains the higher energy loss for smaller immersion depths $\left(\dot{h} \sim \sqrt{120-h_{i}}\right)$. It is also noteworthy to mention that the loss coefficient is higher when the meniscus falls as opposed to rising, meaning that most of the energy dissipated due to kinetic energy occurs during the meniscus fall.

\section{Critical value of $\omega$}

Previously, Masoodi et al. ${ }^{9}$ stated the critical criterion for oscillations in terms of the non-dimensional parameter, $\omega>\omega^{*}$, where $\omega$ is as defined in Eq. (4). They found the critical value $\omega^{*}=0.25$, which we note pertains to the case of $h(0)=0$ in our analysis. However, for our specific experiment, we have $h(0)=h_{f} \gg 0$. In this case, following the analysis in Sec. II, and in particular Eq. (8), the inequality for oscillatory behavior becomes $\omega>(1+\alpha) / 4$, with $\alpha=h_{i} / h_{e}$. Figure 11 plots all of our experimental conditions in dimensionless form of $(1+\alpha) / 4$ versus $\omega$, indicating where oscillations are observed or not.

Taking the example of $70 \%$ glycerol with $R=2 \mathrm{~mm}$ and experimental immersion depths $h_{i}=10$ up to $70 \mathrm{~mm}$, then $(1+\alpha) / 4=0.89-4.73$, with the corresponding value of $\omega$ $=0.96$. As such, we would not expect any oscillations except for the smallest immersion, which is indeed the case-as indicated by the data set highlighted by the red rectangle in Fig. 11 . Additional cases where we do not observe oscillations are for $50 \%, 60 \%$ and $70 \%$ glycerol, with the $2 \mathrm{~mm}$ tube, for which the corresponding values of $\omega$ are $0.53,0.17$, and 0.032 , respectively.

Clearly, we are limited by the spatial resolution of the optical system, which means that if any oscillations were present for any of these conditions, they would have to be on the lengthscale of $50 \mu \mathrm{m}$ or below. As such, for our system, we find that the critical condition, $\omega>\omega^{*}=(1+\alpha) / 4$, identified by the theory in Sec. II accurately describes the physical phenomenon. To our knowledge, this represents the first systematic verification of a critical parameter for meniscus oscillations. 

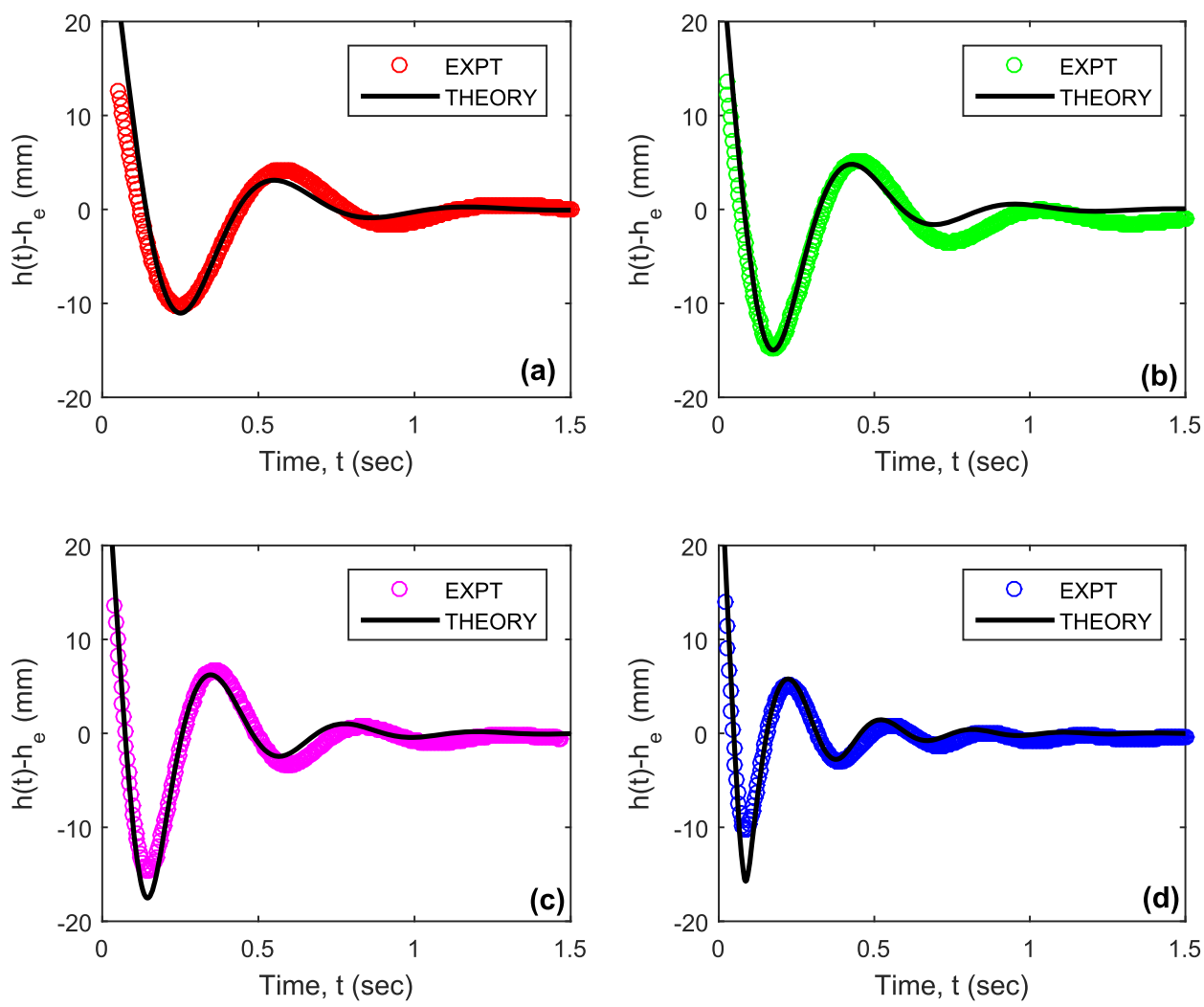

FIG. 7. Comparison between experiment and theory for select cases from Fig. 6 with (a) $h_{i}=66 \mathrm{~mm}$, (b) $h_{i}=46 \mathrm{~mm}$, (c) $h_{i}=27 \mathrm{~mm}$, and (d) $h_{i}=6 \mathrm{~mm}$.

\section{E. Period of oscillation}

In the oscillatory regime, i.e., $\omega>(1+\alpha) / 4$, the phenomenon is best characterized by the half-period of oscillation, $t_{p}$, which is an easily measurable feature across the entire parameter space. Empirically, we find $t_{p}$ can be reasonably well collapsed in terms of either the immersion depth, $h_{i}$, below the free-surface $o r$ in terms of the fill-to-immersion ratio, $h_{f} / h_{i}$. Figure 12 plots the period of oscillation (for water) versus the tube depth where the data points represent the mean value across the video duration and several repeat trials. Note that the period is defined as the time between consecutive local minimums and maximums, as indicated by the inset graphic. The error bars in this data are formed by averaging across both multiple trials and the duration of our observation [i.e., $\mathrm{O}(1-$ $10 \mathrm{~s})$ ] which, given their small size, indicates that the period does not decay as the meniscus converges to the equilibrium. However, the data does show that $t_{p}$ increases gradually as $h_{i}$ increases; For $R \geq 2 \mathrm{~mm}$, we find that $t_{p}$ is virtually independent of $R$ and that $t_{p} \approx 0.1 \mathrm{~s}$ for $h_{i} \approx 6 \mathrm{~mm}$ and $t_{p} \approx 0.3 \mathrm{~s}$ for

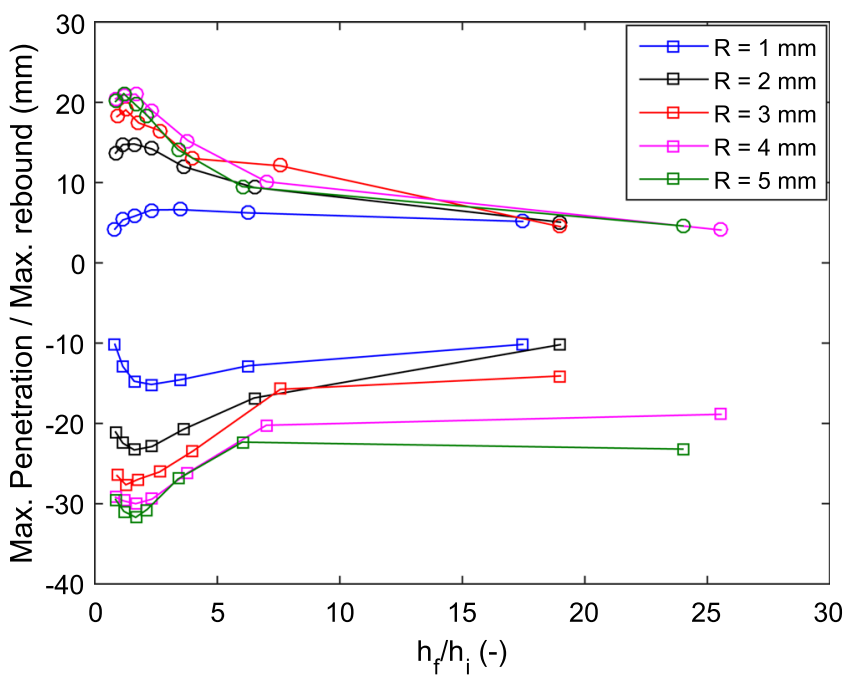

(a)

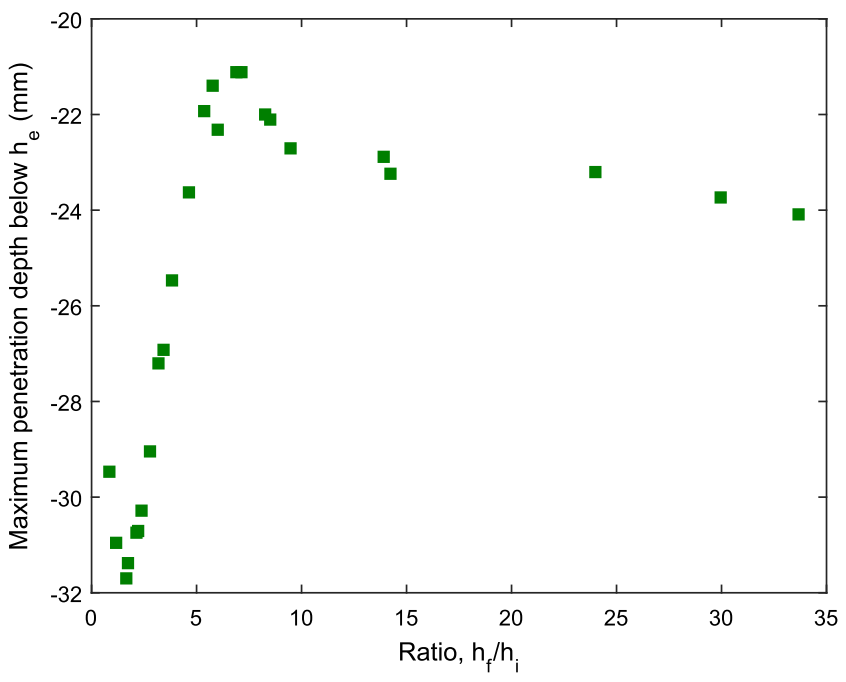

(b)

FIG. 8. (a) Maximum meniscus penetration and maximum rebound heights (relative to $h_{e}$ ) for different tube diameters and fill-to-immersion ratios. Data shown is for water with $R=1,2,3,4$, and $5 \mathrm{~mm}, \omega \approx 18,402,2483,10062,38320$. (b) Maximum penetration depth for water with $R=5 \mathrm{~mm}$, revealing the true local maxima at $h_{f} / h_{i}=1.67$. 


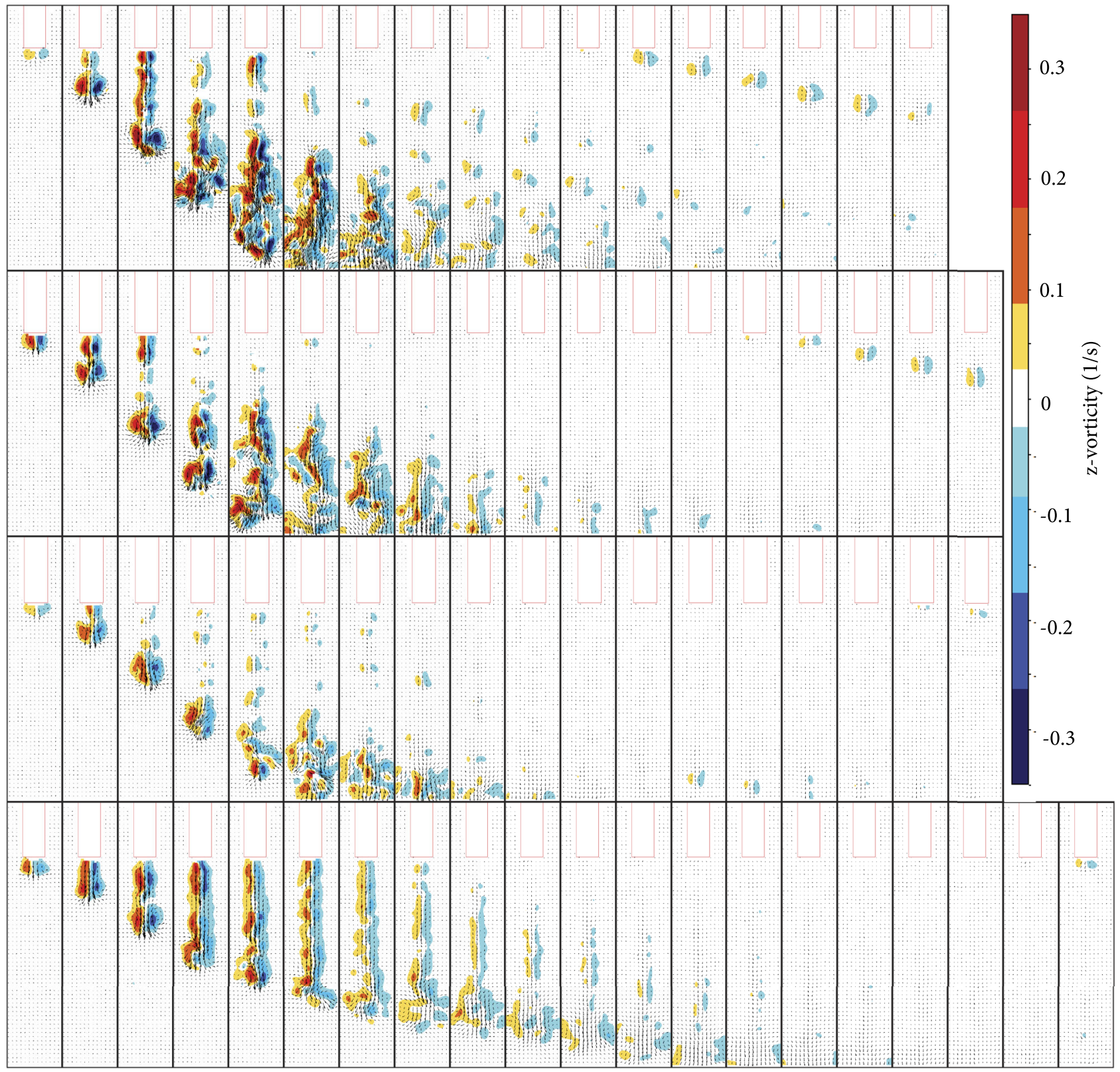

FIG. 9. Vorticity field sequences from PIV recordings of the first rebounds of a water meniscus in a tube of radius $R=1.25 \mathrm{~mm}$. The immersion depths are $h_{i}=10,30,50$, and $70 \mathrm{~mm}$ and the fill heights above the free-surface, $h_{f}=110,90,70$, and $50 \mathrm{~mm}$. Frames are separated by $d t=20 \mathrm{~ms}$. The corresponding values of $\alpha=0.85,2.55,4.26$, and 5.96. Arrows represent the velocity vectors where $1 \mathrm{~m} / \mathrm{s}$ is 10 pixels. Colors represent the $z$-vorticity (1/s). The mask (red box) at the top of each image represents where the straw was in the flow field and the masks are 84 pixels across or 4 mm outside diameter.

$h_{i} \approx 65 \mathrm{~mm}$. For $R=1 \mathrm{~mm}$, the equivalent increase in $t_{p}$ is from approximately 0.15 to $0.35 \mathrm{~s}$.

Before proceeding to examine the remaining data, however, one clear observation to note is that the period of oscillation is consistently higher for the smallest tube with inner diameter of $2 \mathrm{~mm}$ than the other tube diameters. Again, we believe this is due to the fact that the tube diameter is smaller than the capillary length $l_{c}=\sqrt{\sigma /(\rho g)}=2.7 \mathrm{~mm}$ for water; thus both surface tension forces and viscous forces play an increasingly significant role, where the surface tension and viscous terms in Eq. (2) scale as $\sim 1 / R$ and $\sim 1 / R^{2}$, respectively. We can only expect viscous dissipation (due to wall effects) to be negligible for timescales significantly smaller than $\tau_{\mu}=\rho R^{2} / \mu$, which is not the case for
$R=1 \mathrm{~mm}$, where $\tau_{\mu}=1 \mathrm{~s}$. Therefore, the failure of the complete collapse of the data for oscillation period is an indication that surface tension and viscous forces are at play for $2 R<l_{c}$.

The trends observed in the experimental data are qualitatively well-described by the theory, i.e., Eq. (11). The solution to this equation is represented by the dashed lines in Fig. 12. The largest absolute discrepancy between theory and experiment is found for the smallest radius. In particular, we find that the solution for $R=1 \mathrm{~mm}$ underestimates the data substantially for the deepest immersion depths, for example the discrepancy between the experiment and Eq. (11) is approximately $25 \%$ for $h_{i}=65 \mathrm{~mm}$, but this difference diminishes as $h_{i}$ decreases, so that the theory overlaps the lower error bar at 


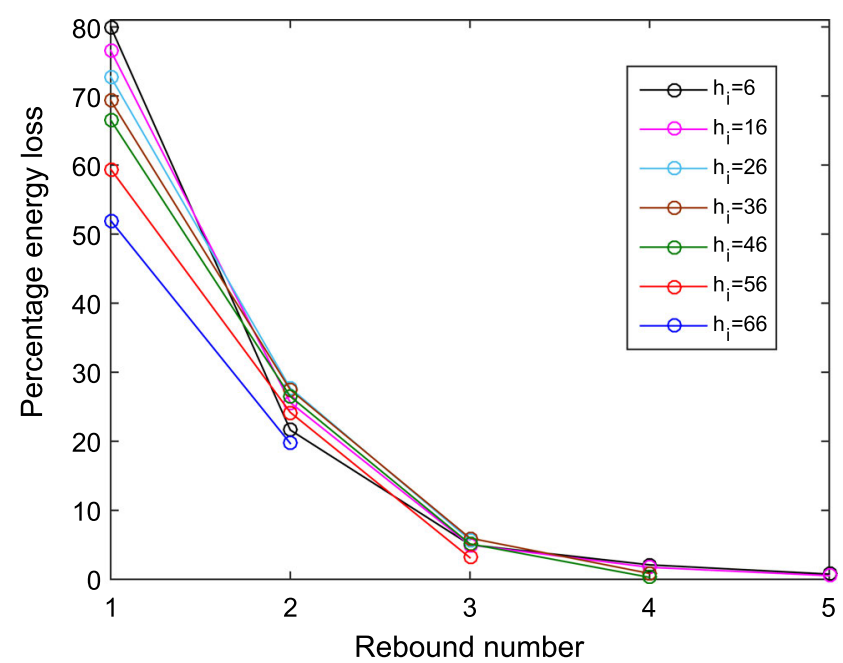

FIG. 10. Percentage energy loss at successive rebounds. The data shows the case for water in a $R=1 \mathrm{~mm}$ tube, which corresponds to the data in Fig. 6.

$h_{i}=6 \mathrm{~mm}$. For the larger tube diameters, the discrepancy is significantly smaller and the theory provides a good quantitative match of the period of oscillation, again indicating that our analytical model in Sec. II is capable of capturing the salient features of this phenomenon.

One striking observation across the entire span of our data is the lack of dependence on viscosity, which can be seen in Fig. 13. This can be partly rationalized by considering Eq. (11), which gives the period of oscillation in terms of $\omega, h_{e}$ and $\alpha$. In the limit of large $\omega$, we have for the demonimator $\sqrt{g \omega} \sqrt{4 \omega-(1+\alpha)} \sim 2 \omega \sqrt{g}$, therefore we can give $t_{p} \approx \pi \sqrt{\left(h_{e}+h_{i}\right) / g}$, which for small $h_{e}$ means that $t_{p} \sim \sqrt{h_{i}}$. Combining all data with $\omega \gtrsim O(10)$, we find that indeed this square-root dependence on the immersion depth is valid, as shown in Fig. 13.

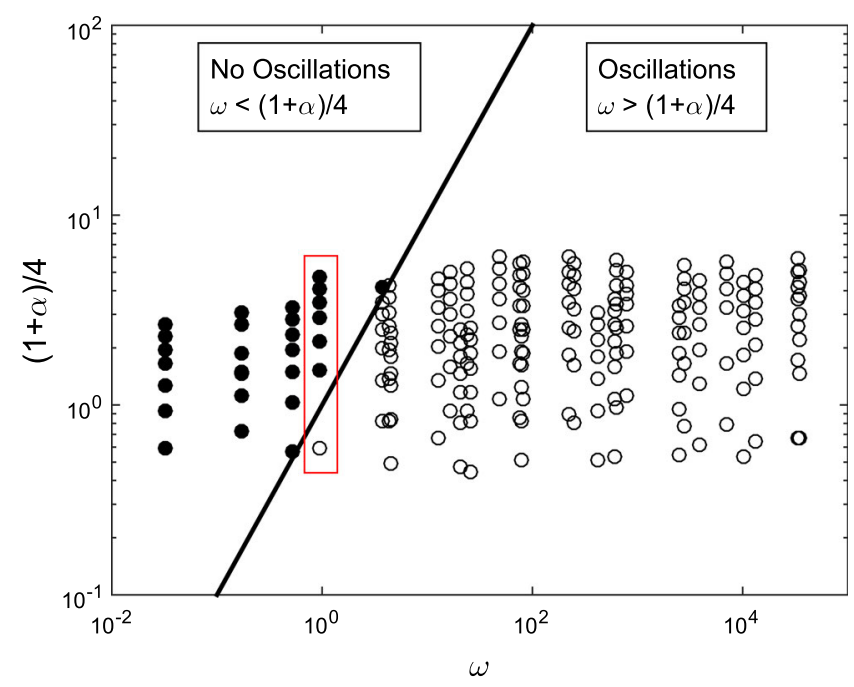

FIG. 11. Plot of all 210 experimental conditions in the dimensionless form of $(1+\alpha) / 4$ versus $\omega$. The thick black line indicates parity, with the data points to the right satisfying $\omega>(1+\alpha) / 4$. The open symbols indicate where oscillations were observed and solid symbols indicate where no oscillations occurred. The data in the red rectangle are for $70 \%$ glycerol with $R=2 \mathrm{~mm}$, discussed in the main text.

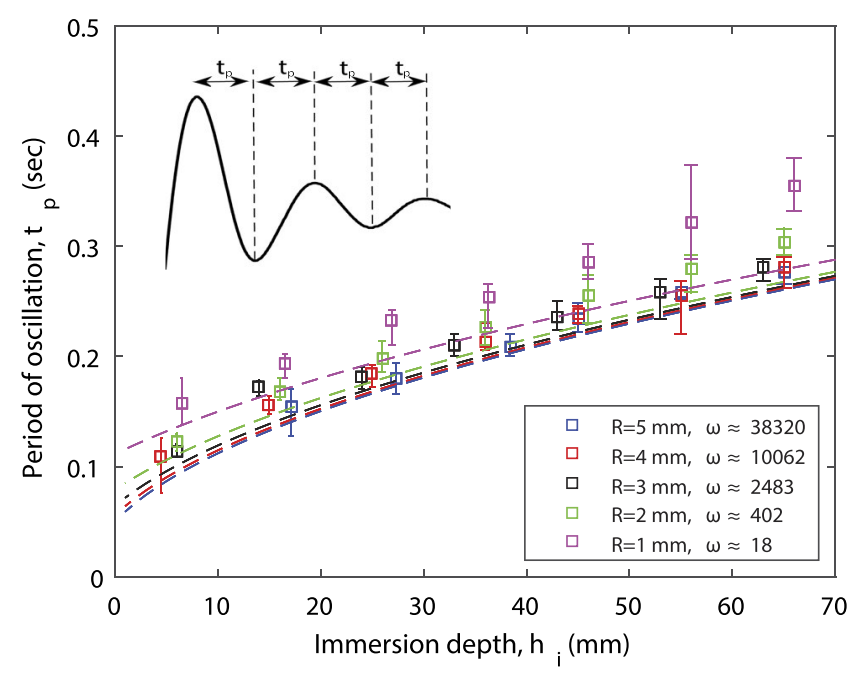

FIG. 12. Half-eriod of oscillation, $t_{p}$, versus immersion depth, $h_{i}$, for all tube radii for water corresponding to values of $\omega \approx 18402,2483,10062,38320$. The inset graphic depicts the measurement of the period. The dashed lines represent the solution to equation 2.7 for the different tube radii (colors match the experimental data symbols).

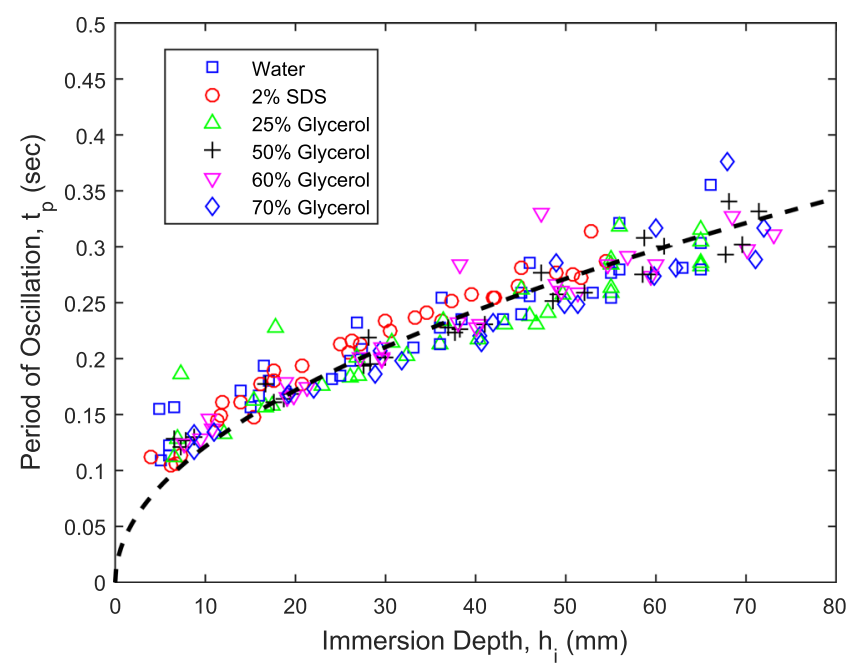

FIG. 13. Period of oscillation versus immersion depth for all fluids and tube sizes. Each data point represents the mean value of several repeat trials. The dashed line indicates the best fit to the data with $t_{p} \propto \sqrt{h_{i}}\left(r^{2}=0.89\right)$.

\section{JET FORMATION}

A striking feature during the very first rebound is the formation of a jet when the meniscus reaches its minimum depth below the equilibrium level. In some cases, for shallow immersion, the meniscus drops below the end of the tube and creates an air cavity, as shown in Fig. 14(a). In this particular case, hydrostatic pressure acts to pinch the air cavity, which appears qualitatively reminiscent of the air cavity formed by a plunging disk (Ref. 1). When the pinch-off occurs (between images 6 and 7 in this sequence), an air bubble is created along with two jets - one that shoots up into the tube and one into the bubble itself (seen in image 8). The initial speed of the jet in this instance is $4.2 \mathrm{~m} / \mathrm{s}$. As the immersion depth is increased [Figs. 14(b)-14(d)], the jet speed changes. In Fig. 14(b), the meniscus protrudes slightly from the tip of the tube but no pinch-off or bubble occurs, instead we observe an intricate 


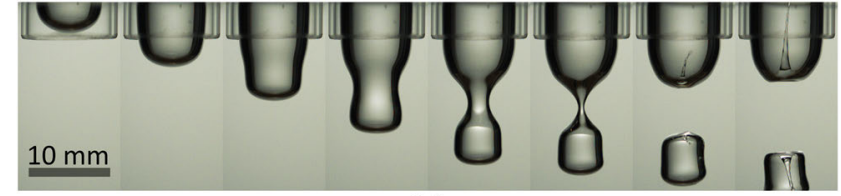

(a)

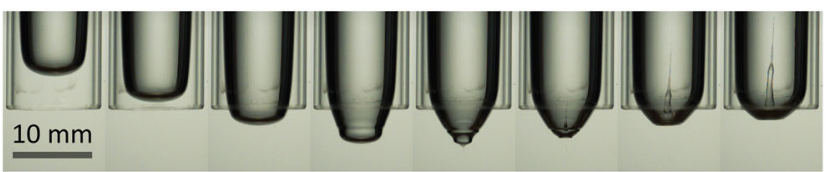

(b)

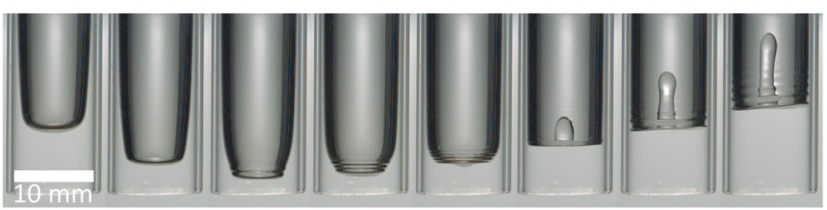

(c)

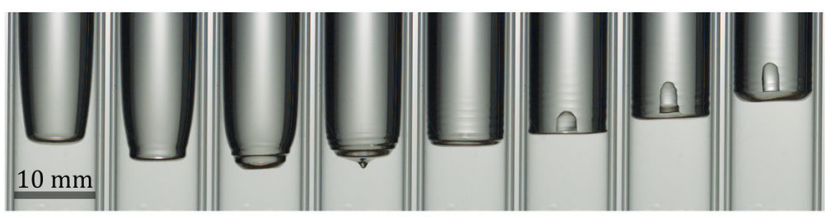

(d)

FIG. 14. Meniscus evolution near the maximum penetration depth for (a) $h_{f}$ $=115 \mathrm{~mm}, h_{i}=5 \mathrm{~mm}$, (b) $h_{f}=103 \mathrm{~mm}, h_{i}=17 \mathrm{~mm}$, (c) $h_{f}=93 \mathrm{~mm}$, $h_{i}=27 \mathrm{~mm}$, (d) $h_{f}=82 \mathrm{~mm}, h_{i}=38 \mathrm{~mm}$. The times relative to pinch-off/jet start are (a) $t=-16.3,-12.7,-9,-5.2,-1.5,-0.25,1,3.5 \mathrm{~ms}$; (b) $t=-15$, $-11.4,-7.6,-3.8,-0.12,0.5,2.8,3.6 \mathrm{~ms}$; (c) $t=-17.1,-9.6,-2.1,1.6,5.4$, $12.8,20.4,27.8 \mathrm{~ms}$; (d) $t=-18.75,-12.5,-6.25,0,6.25,12.5,18.75,25 \mathrm{~ms}$.

wave focusing process that leads to a faster jet with an initial tip speed of $V_{j e t} \approx 10.68 \mathrm{~m} / \mathrm{s}$ in this realization. As the immersion depth increases past $h_{i}=23 \mathrm{~mm}$, the meniscus no longer escapes the end of the tube and the jet speed is dramatically reduced to $V_{\text {jet }} \approx 0.96 \mathrm{~m} / \mathrm{s}$ and $0.54 \mathrm{~m} / \mathrm{s}$ in Figs. 14(c) and 14(d), respectively. The jet becomes notably thicker in such cases and further increasing the immersion depth leads to complete suppression of the jet. The immersion depth at which the jet is completely suppressed coincides precisely with the maximum penetration depth (relative to the equilibrium capillary rise height) and the maximum rebound height. This is quite intuitive from an energy perspective and also helps to explain discrepancies between the theoretical solution and the experiments since energy dissipation due to jetting is not accounted for in the model.

The details of the wave focusing process for Fig. 14(b) are shown in Figs. 15(a)-15(c). The fact that multiple waves are present at the tip leads to an interesting observationnamely - a dual structure jet formation, shown by the image sequence in Fig. 15(c). Here, we can clearly observe a highspeed and slender tip which precedes a thicker base. This dual-structure jet formation has been reported previously for jets created by the collapse of voids in granular beds (Refs. 8 and 11). In the present study, the dual-structure jet appears to be a consequence of the wave focusing; The fine tip is the product of the inertial focusing of the pointed end in image 8 in Fig. 15(b), then as the fluid is channeled into the jet, a sudden expansion occurs when the crest of the

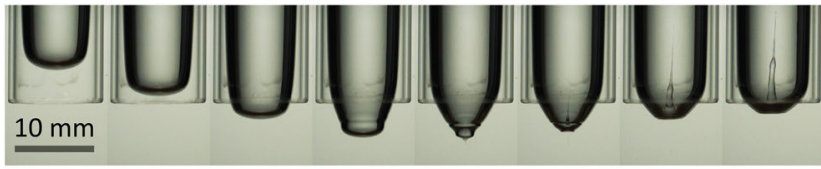

(a)

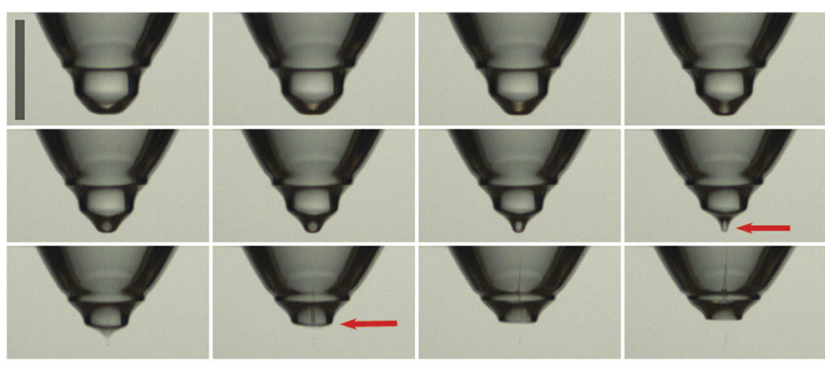

(b)
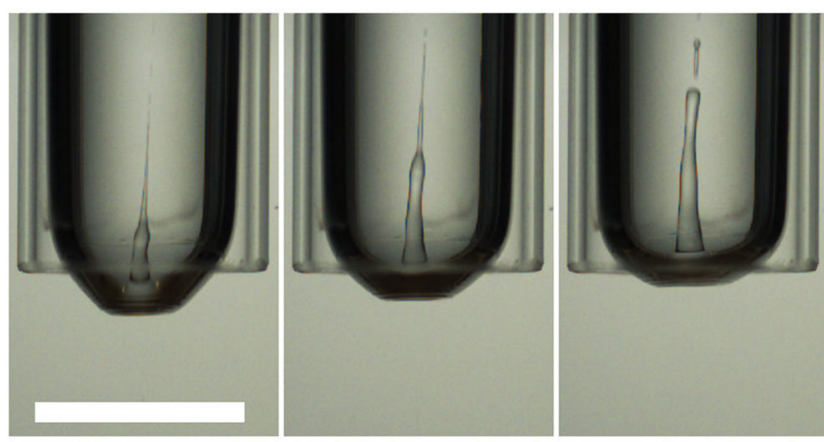

(c)

FIG. 15. Jet formation near the maximum penetration depth for water in a tube with radius $R=5 \mathrm{~mm} . h_{f}=103 \mathrm{~mm}, h_{i}=17 \mathrm{~mm}$ corresponding to the same realisation as Fig. 14(b). (a) Overview of the meniscus penetration and rebound below the end of the tube, (b) Close-ups of the wave focusing between frames 4 and 6 in panel (a). The red arrows correspond to the frames just prior to direction reversal and first jet motions, respectively. The scale bar is $5 \mathrm{~mm}$ long. (c) Close-ups of the dual-structure appearance of the jet. The scale bar is $10 \mathrm{~mm}$ long. In (a), the times relative to reversal are $t=-15,-11.4,-7.6$, $-3.8,-0.12,0.5,2.8,3.6 \mathrm{~ms}$; In (b), the frames are separated by $d t=125 \mu \mathrm{s}$; In (c) the frames are separated by $d t=1.25 \mathrm{~ms}$. See also video 2 of the supplementary material accompanying this figure.

next wave is met, as indicated by the red arrow in image 10 of this sequence. The fine tip breaks up into droplets and leaves just the thicker jet to proceed up into the tube, as shown in Fig. 15(c).

By calculating the jet speed over a number of realizations, we observe that there is a distinct peak in the jet speed, $V_{j}$, which varies as a function of immersion depth, $h_{i}$. The qualitative trend is similar for all fluids where the jet is observednamely-an increase in jet speed as the immersion depth increases for $h_{i} / R \lesssim 2.5$, after which a dramatic drop in jet speed occurs. The maximum jet speeds observed were $V_{\text {jet } \text {, max }}$ $=13.66 \mathrm{~m} / \mathrm{s}$ for SDS (at $h_{i} / R=2.36$ ), $V_{\text {jet } \text {, } \operatorname{sax}}=12.9 \mathrm{~m} / \mathrm{s}$ for water $\left(\right.$ at $h_{i} / R=2.26$ ) and $V_{\text {jet } \max }=5.4 \mathrm{~m} / \mathrm{s}$ for $25 \%$ glycerol (at $\left.h_{i} / R=1.06\right)$.

The overall trend is best captured across the entire parameter space by plotting the normalized jet speed, $V_{j} / \sqrt{g h_{i}}$ against the normalized immersion depth, $h_{i} / R$, as shown in Fig. 16 . The quantity $\sqrt{g h_{i}}$ physically represents the gravitational-inertial velocity, which was used previously to scale jet speeds arising from free-surface collapse (e.g., Ref. 4). We find that for $h_{i} / R \lesssim 2.5$, the normalized jet speed is virtually independent 


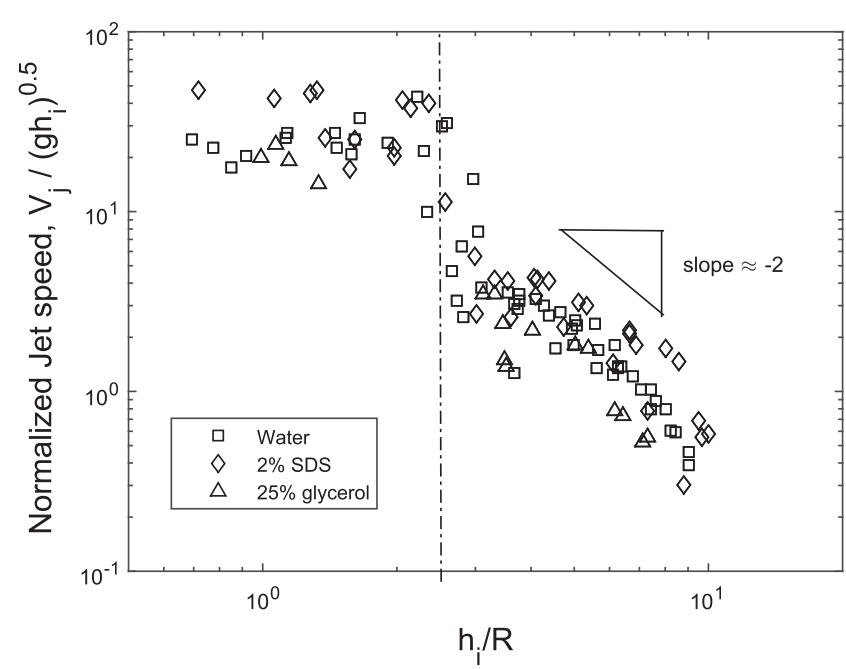

FIG. 16. Normalized jet speed, $V_{j} / \sqrt{g h_{i}}$, plotted against the non-dimensional immersion depth of the tube, $h_{i} / R$. The vertical dashed line indicates $h_{i} / R$ $\approx 2.5$, after which a dramatic drop in in jet speed occurs. Data for all observed jets is included.

of the immersion depth, but for $h_{i} / R \gtrsim 2.5$, there is a sharp decrease until the jet is completely suppressed, which could be approximated by the relation $V_{j} / \sqrt{g h_{i}} \sim\left(h_{i} / R\right)^{-2}$.

The high-speed jets for $h_{i} / R \lesssim 2.5$ are due to the cavity pinch-off, driven by hydrostatic pressure, whilst the dramatic reduction in speed is explained by the suppression of the air cavity pinch-off. Furthermore, as the immersion depth increases, the meniscus fails to protrude from the end of the tube (see Fig. 9), isolating it from the bulk liquid and hydrostatic effects. Eventually, the jet is completely suppressed, which occurs earlier (lower values of $h_{i} / R$ ) in narrow tubes and for more viscous fluids; for example with $25 \%$ glycerol, the jet ceases for $h_{i} / R \gtrsim 7$, whilst for water and $2 \%$ SDS, the jet does not disappear until after $h_{i} / R \gtrsim 10$. In contrast, we did not observe jets for 50,60 or $70 \%$ with the current parameter space.

\section{CONCLUSIONS}

In conclusion, we have conducted a thorough and systematic study of the motion of a falling column of liquid, bounded in a glass tube. The column started at a height above the equilibrium capillary rise height and, depending on the tube radius and fluid properties, the meniscus either decayed to the equilibrium or entered an oscillatory regime. The principal experimental measurement taken was the location of the meniscus, which was easily tracked from high-speed video recordings and converted into characteristics such as energy loss and period of oscillation.

Our simple analytical model of this phenomenon stemmed from a force balance, including capillary and viscous effects, previously considered by Refs. 9 and 7, with the principal difference being that the column starts from far above equilibrium. The two non-dimensional parameters in this model were the scaled immersion depth, $\alpha=h_{i} / h_{e}$, and a parameter incorporating fluid properties and tube radius, $\omega=\frac{\rho^{3} g^{2} R^{5}}{128 \sigma \mu^{2}}$. From an approximate analytical solution to the main equation identified, we identified a critical condition for the oscillatory regime, given as $\omega>(1+\alpha) / 4$, which agreed very well with the experimental observations. This key result differs from previous works on capillary rise dynamics since our framework includes the non-zero initial height.

The oscillatory regime was characterized by the halfperiod of oscillation (between consecutive maxima and minima), which was measured and found to be a function of the immersion depth (and initial height of the meniscus), but independent of fluid viscosity. The independence of this period on fluid properties could be explained by considering the theoretically-derived period of oscillation, which gave $t_{p} \approx \pi \sqrt{\left(h_{e}+h_{i}\right) / g}$.

One striking local feature in this phenomenon was the formation of a jet, which occurred during the first rebound, but only for the lowest viscosity fluids with $\mu<2.3 \mathrm{mPa} \mathrm{s}$. The highest jets speeds, up to $\sim 13 \mathrm{~m} / \mathrm{s}$, were observed for the case when the meniscus actually protruded from the end of the tube. In such cases, the jet is produced from the hydrostaticallydriven pinch-off of the air cavity. In contrast, when the meniscus did not protrude from the tube, the jet speed was significantly lower. In either case, this jet serves as an energy dissipation mechanism that is not currently accounted for in the theory.

Future extensions of this work might consider a liquidliquid system, which would add additional damping effects and complex contact line dynamics at the meniscus.

\section{SUPPLEMENTARY MATERIAL}

See supplementary material for videos 1 and 2 .

Video 1 (Fig. 1): This video shows the motion of a water meniscus in a glass tube with inner diameter of $6 \mathrm{~mm}$. The initial height above the surface was $97 \mathrm{~mm}$ and the immersion depth of the tube is $23 \mathrm{~mm}$. The original video was captured at $500 \mathrm{fps}$, whilst the playback speed is $30 \mathrm{fps}$.

Video 2 (Fig. 15): This video shows the fine wavefocusing process that leads to the formation of a fast liquid jet. Note that the meniscus actually falls below the end of the tube, creating an air cavity prior to the reversal of direction. The original video was captured at $8000 \mathrm{fps}$, whilst the playback speed is $30 \mathrm{fps}$.

${ }^{1}$ R. Bergmann, D. van der Meer, S. Gekle, A. van der Bos, and D. Lohse, "Controlled impact of a disk on a water surface: Cavity dynamics," J. Fluid Mech. 633, 381-409 (2009).

${ }^{2}$ H. Cao, C. Amador, X. Jia, and Y. Ding, "Capillary dynamics of water/ethanol mixtures," Ind. Eng. Chem. Res. 54, 12196-12203 (2015).

${ }^{3}$ S. Das and S. K. Mitra, "Different regimes in vertical capillary filling," Phys. Rev. E 87, 063005 (2013).

${ }^{4}$ E. Ghabache, T. Seon, and A. Antkowiak, "Liquid jet eruption from hollow relaxation," J. Fluid Mech. 761, 206-219. (2014).

${ }^{5}$ S.-L. Lee and H.-D. Lee, "Evolution of liquid meniscus shape in a capillary tube," J. Fluids Eng. 129, 957-965 (2007).

${ }^{6}$ S. Levine, J. Lowndes, E. J. Watson, and G. Neale, "A theory of capillary rise of a liquid in a vertical cylindrical tube and in a parallel-plate channel," J. Colloid Interface Sci. 73, 136-151 (1980).

${ }^{7}$ E. Lorenceau, D. Quere, J.-Y. Ollitraut, and C. Clanet, "Gravitational oscillations of a liquid column in a pipe," Phys. Fluids 14(6), 1985-1992 (2002).

${ }^{8}$ J. O. Marston, J. P. K. Seville, Y.-V. Cheun, A. Ingram, S. P. Decent, and M. J. H. Simmons, "Effect of packing fraction on granular jetting from solid sphere entry into aerated and fluidized beds" Phys. Fluids 20, 023301 (2008). 
${ }^{9}$ R. Masoodi, E. Languri, and A. Ostadhossein, "Dynamics of liquid rise in a vertical capillary tube," J. Colloid Interface Sci. 389, 268-272 (2013).

${ }^{10}$ D. Quere, E. Raphael, and J.-Y. Ollitrault, "Rebounds in a capillary tube," Langmuir 15, 3679-3682 (1999).

${ }^{11}$ J. R. Royer, E. I. Corwin, A. Flior, M.-L. Cordero, M. L. Rivers, P. J. Eng, and H. M. Jaeger, "Formation of granular jets observed by high-speed X-ray radiography," Nat. Phys. 1, 164 (2005).
${ }^{12}$ H. E. Schulz and D. Z. Zhu, "Oscillations of water levels in vertical semiimmersed tubes: Analytical solutions and experimental verification," J. Appl. Fluid Mech. 10(6), 1515-1525 (2017).

${ }^{13} \mathrm{P}$. Wu, H. Zhang, A. Nikolov, and D. Wasan, "Rise of the main meniscus in rectangular capillaries: Experiments and modeling," J. Colloid Interface Sci. 461, 195-202 (2016).

${ }^{14}$ B. V. Zhmud, F. Tiberg, and K. Hallstensson, "Dynamics of capillary rise," J. Colloid Interface Sci. 228, 263-269 (2000). 